\title{
A REALIDADE DO IDOSO INSTITUCIONALIZADO FRENTE À VISITA FAMILIAR: UM ESTUDO QUANTITATIVO
}

FARIA, Aline Cristina de. Graduação em Enfermagem pelo Centro Universitário de Araraquara. Especialista em Saúde da Família pela Unifesp. Enfermeira gestora da atenção básica na Prefeitura Municipal de Araraquara. Av. José Cendon Farto, 127. Selmi Dey 4 - Araraquara-SP. Tel: (16) 99722-0065/(16) 3324-2511.

E-mail: alinecrfaria@hotmail.com.

ANTONIO, Sandra Aparecida Emidio. Graduação em Enfermagem pelo Centro Universitário de Araraquara. Especialista em urgência e emergência e cuidados intensivos. Enfermeira do Hospital Beneficência Portuguesa de Araraquara. Rua Pastor Antonio da Silva Cortejo, bl 1D, apt 112-D. Vila Biagioni - Araraquara-SP.

EBISUI, Cássia Tiêmi Nagasawa. Profa. Dra. do curso de Enfermagem do Centro Universitário de Araraquara e coordenadora pedagógica do Centro Estadual de Educação Tecnológica Paula Souza. Av. Gertrudes Leite de Souza Pinto, 861. Vila Xavier - Araraquara-SP.

\section{RESUMO}

O envelhecimento para o indivíduo consiste na perda gradativa de seus papéis sociais e o vazio experimentado por não encontrar novas funções. Esses sentimentos podem levá-lo ao sofrimento psíquico, principalmente quando há falhas na integração familiar. Este estudo quantitativo objetivou identificar o número de idosos institucionalizados que recebe visitas, assim como sua frequência e o vínculo dos visitantes, em um município do interior do estado de São Paulo. Para a coleta de dados utilizou-se questionário estruturado contendo questões de interesse ao estudo para que os 47 idosos $(100 \%)$ institucionalizados respondessem. Os resultados mostram que, dentre os sujeitos respondentes, $64 \%$ dos idosos entrevistados recebiam visitas; em relação à frequência dessas visitas, $26 \%$ referem receber visitas no período superior a um mês. Os achados revelam ainda que $63 \%$ dos visitantes foram identificados como outros parentes (sobrinhos, irmãos, netos e primos e amigos) e, destes, 47\% possuíam filhos que não visitavam seus pais. Concluiu-se que o achado de maior relevância neste estudo, além da corroboração da multiplicidade de perdas presentes na história de vida dos idosos institucionalizados, foi o sentimento de abandono inerente a todos esses idosos em relação aos filhos e outros parentes, assim como pela sociedade em geral, havendo necessidade de valorização dessa população emergente tanto nas instâncias governamentais como familiares.

Palavras-chave: Idoso; Instituição de longa permanência para idosos; Institucionalização; Família.

\section{THE REALITY OF INSTITUTIONALIZED ELDERS CONSIDERING RELATIVES' VISIT: A QUANTITATIVE STUDY}

\section{Abstract}

Ageing for the individual consists of the gradual loss of his/her social roles and the emptiness experienced by not finding new roles. These feelings can lead to psychic suffering especially when there are failures in family integration. This quantitative study aimed at identifying the number of institutionalized elderly receiving visits, as well as their frequency and the link of the visitors, in a municipality in the State of São Paulo, Brazil. Data were collected by asking the $47(100 \%)$ institutionalized elderly to answer a structured questionnaire containing questions of interest to the study. The results show that among the interviewed elderly, $64 \%$ received visits; considering the frequency of these visits, $26 \%$ said that received visits in the period exceeding 1 month. The findings reveal that $63 \%$ of the visitors were other relatives (brothers, grandchildren and nephews, 
cousins and friends), among them, $47 \%$ had children who didn't visit their parents. We concluded that the most relevant finding in this study, in addition to the corroboration of the multiplicity of these losses in the history of life of institutionalized elderly, was the sense of abandonment, inherent in all these elderly people, by their sons, daughters and other relatives, as well as by society in general, showing the need to value this emerging population both in governmental instances as well as in their families.

KEYwORDS: Elder; Long stay institution for elders; Institutionalization; Family.

\section{INTRODUÇÃo}

O crescimento da população idosa é um fenômeno de impacto mundial, tendo como causas a diminuição da mortalidade e fecundidade, além do aumento da expectativa de vida. Pessoas acima de 60 anos eram habitualmente encontradas nos países desenvolvidos; atualmente, o envelhecimento populacional é um fenômeno que está ocorrendo nos países em desenvolvimento. O que os difere é que, enquanto nos primeiros essa transição foi lenta e gradual, no segundo grupo essa mudança foi brusca e o processo de transição demográfica tem acontecido graças aos avanços tecnológicos e científicos. Paralelamente a esses fatos, a população passou a ter acesso a vacinas, antibióticos, serviços de saúde, e saneamento básico, que contribuíram para adoção de medidas de prevenção e cura de muitas doenças, promovendo uma melhora na qualidade de vida e um crescente aumento da longevidade da população (REIS et al., 2008).

A velhice deveria ser enfrentada como mais uma etapa da vida, que pode e deve ser significativa. Existem idosos que agem assim e atingemum equilíbrio imenso, mas a maioria busca um novo sentido para a sua vida. A situação familiar do idoso no Brasil reflete o efeito cumulativo em eventos socioeconômicos, demográficos e de saúde ao longo dos anos, demonstrando que o tamanho da prole, as separações, o celibato, a mortalidade, a viuvez, os recasamentos e as migrações vão originando, no desenvolver das décadas, tipos de arranjos familiares e domésticos, em que o morar sozinho, com parentes ou em asilos, pode ser o resultado desses desenlaces (PAPALEO, 2005).

Para minimizar os efeitos psicológicos ocasionados pela institucionalização, esses locais que acolhem a pessoa idosa devem seguir o rigor de seu funcionamento, conforme legislações existentes.

Em 26 de setembro de 2005, foi adotada a Resolução da Diretoria Colegiada - RDC 283 da Agência Nacional de Vigilância Sanitária - ANVISA, que define as normas para funcionamento adequado das Instituições de Longa Permanência para Idosos ILPIs, abrangendo os seguintes aspectos: organização, recursos humanos, característica física, alimentação, limpeza e plano de atenção à saúde dos idosos residentes. Ainda de acordo com a RDC, serão denominadas instituições específicas para idosos todos os estabelecimentos que disponibilizarem profissionais capacitados, suportes físicos adequados e que conseguirem realizar tarefas normais do cotidiano para receber indivíduos com idade igual ou superior a 60 anos, por longa ou curta estadia, de forma gratuita ou particular, garantindo condições de liberdade, dignidade e cidadania (BRASIL, 2005).

Popularmente, asilo continua a ser a denominação mais comum para instituições destinadas aos idosos. Ainda hoje, existe um grande número de instituições de caráter asilar, com função basicamente assistencial (BORN; BOECHAT, 2006).

Nos debates iniciados há alguns anos nas comissões especializadas da Sociedade Brasileira de Geriatria e Gerontologia - SBGG, em congressos e jornadas, começou a ser adotada a denominação Instituição de Longa Permanência para Idosos - ILPI como correspondente a Long-Term Care Institution, embora a tradução mais correta fosse Instituição de Cuidado de Longo Prazo (BORN; BOECHAT, 2006).

Mesmo com a implantação de leis para assegurar um local adequado para a pessoa idosa, inúmeros trabalhos versam acerca de instituições de longa permanência, porém, apontam que tais locais se caracterizam como um locus negativo para ser um espaço de moradia para idosos. Esses locais 
predispõem a segregação, pois os idosos são afastados de seu ambiente social e humano que sempre frequentaram. Além disso, apresentam normatizações das atividades diárias, uniformização e disciplina que, muitas vezes, não permitem ao indivíduo preservar sua singularidade e seu espaço (PERLINI; LEITE; FURINI, 2007).

A institucionalização é um processo irreversível, em que parte da população idosa precisará se submeter nas próximas décadas. Esse processo pode ser humanizado e funcionar em moldes mais abertos, para que as instituições de longa permanência de cuidado aos idosos não signifiquem apenas depósitos de velhos (PAVAN; MENEGHEL; JUNGES, 2008).

É a crise que afeta a humanidade pela falta de cuidado. Para sair dessa crise, é necessária uma nova ética, que deve nascer de algo essencial ao ser humano, residindo mais no cuidado do que na razão e na vontade. Nesse caso, cuidar é mais que um ato, é uma atitude de preocupação, de responsabilidade e de envolvimento afetivo com o outro. Semamor, o homem não possui o cuidado (BOFF, 1999).

Diante dessa perspectiva, a solidão, o abandono e a falta de atenção são aspectos negativos que podem contribuir para a invisibilidade da pessoa idosa frente aos seus familiares. Com essa preocupação, o objetivo desta pesquisa se constituiu em identificar o número de idosos institucionalizados que possuem filhos, o número de filhos, a cidade de origem antes da institucionalização, se recebem visitas, a frequência das visitas e vínculo dos visitantes, em Instituições de Longa Permanência para Idosos de um município do interior do estado de São Paulo.

\section{Metodologia}

Trata-se de um estudo descritivo de cunho quantitativo.

Em relação ao campo de estudo, foi realizado em Instituições de Longa Permanência para Idosos ILPIs, em um município do interior paulista.

Elegeu-se como público alvo idosos residentes nas ILPIs, que constituíram um universo de 47 idosos institucionalizados.
Foram selecionados para os critérios de inclusão:

- Instituições cadastradas junto à Vigilância Sanitária do município;

- Autorização do responsável pela ILPemparticipar voluntariamente do estudo;

- Idosos residentes em ILPIs, que aceitaram a participar voluntariamente do estudo.

E para os critérios de exclusão:

- Responsáveis e/ou profissional designado pelo responsável pelas ILPs que não aceitarem participar do estudo;

- Idosos que não aceitaram participar da pesquisa, ou que apresentaram incapacidade mental e intelectual para compreender e comunicar-se.

No que tange à coleta de dados, inicialmente foi realizado contato com o administrador das ILPIs escolhidas, explicando os objetivos do estudo e solicitando a autorização para iniciar a pesquisa. Após a aprovação, foi agendado com o responsável pelas instituições uma visita técnica monitorada para aproximação e vínculo com os idosos. Posteriormente, obteve-se uma listagem das pessoas idosas residentes na instituição para possível aproximação das pesquisadoras com os eventuais participantes. Cada idoso, após receber explicações e concordar com a sua participação no trabalho, foi convidado a responder a uminstrumento para coleta de dados, após assinarem o termo de consentimento livre e esclarecido, atendendo à resolução 196/96. O protocolo constituiuse em um questionário estruturado, elaborado para obtenção dos dados de interesse para o estudo.

O projeto foi aprovado pelo Comitê de Ética em Pesquisa do Centro Universitário de Araraquara, sob o protocolo n. ${ }^{\circ}$ 1242/11.

Para análise dos dados foi realizada estatística quantitativa de percentagem, correspondente aos dados levantados com apresentações em gráficos e tabelas. No processo de digitação, optou-se por uma validação via dupla digitação. Os resultados foram analisados através de técnica quantitativa, análise descritiva e crítica reflexiva e, por fim, a conclusão de acordo com os resultados encontrados na pesquisa. 


\section{Resultados e Discussão}

Segundo dados do Instituto Brasileiro de Geografia e Estatística - IBGE (2011), a população do município pesquisado atualmente é de 208.725 mil habitantes, sendo 12.274 homens com mais de 60 anos e 16.500 mulheres com mais de 60 anos, representando uma população de idosos de $13,78 \%$



Distribuição dos idosos que possuem filhos (\%)
(IBGE, 2011).

De acordo com os critérios de inclusão e exclusão estabelecidos, do universo de 210 (100\%) idosos, 47 $(22 \%)$ se enquadraram na pesquisa e tiveram condições de responder ao instrumento de coleta de dados. Assim, os 47 idosos constituíram a amostra efetiva deste estudo.

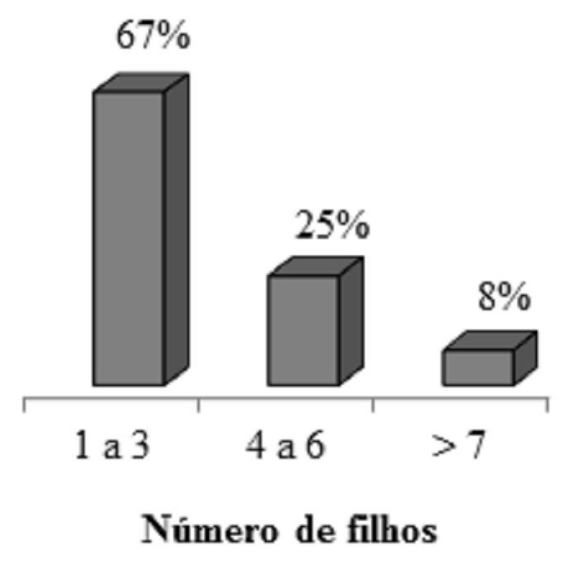

Figura 1 - Distribuição percentual dos idosos que possuem filhos e número de filhos. Araraquara, 2011.

Fonte: Dados de pesquisa.

Em relação ao número de filhos, $51 \%(\mathrm{n}=24)$ dos entrevistados possuem filhos, enquanto $49 \%(n=23)$ dos entrevistados afirmaram não terem filhos. Dentre os que afirmaram ter filhos, $67 \%(n=16)$ possuem de 1 a 3 filhos, $25 \%(n=06)$, de 4 a 6 filhos e $8 \%(n=2)$, mais que 7 filhos.

Considerando os dados apresentados, percebe-se que o fato de os idosos possuírem ou não filhos pode não ter influenciado em sua institucionalização.

No Brasil, o suporte provido pela família, base principal do apoio oferecido ao idoso, como ocorre na maioria dos países em desenvolvimento, enfrentam dificuldades, como: a não existência de políticas sociais de suporte aos cuidadores; o tamanho das famílias no Brasil, que vêm diminuindo devido à queda da fecundidade; o aumento na proporção de separações conjugais; idosos residindo com familiares cuja renda total não atinge três salários mínimos; e o sistema de suporte formal que não substitui o papel da família (CHAIMOWICZ, 1998).

A sociedade brasileira vem passando por importantes alterações em sua estrutura. Há algumas décadas está ocorrendo uma permanente queda da hegemonia do modelo de família formada por casal com filhos, o que corrobora comos dados encontrados (PINHEIRO; GALIZA; FONTOURA, 2009). 
Cidade de origem antes da institucionalização (\%)

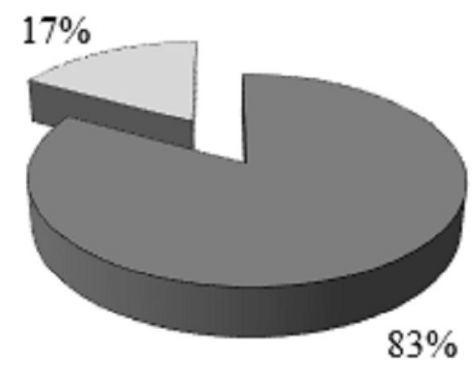

$\square$ Araraquara

$\square$ Outras

Figura 2 - Distribuição percentual dos idosos entrevistados segundo a cidade de origem antes da institucionalização. Araraquara, 2011.

Fonte: Dados de pesquisa.

Quanto à cidade de origem antes da institucionalização, $83 \%(\mathrm{n}=39)$ residiam no município pesquisado e $17 \%$ $(\mathrm{n}=08)$ migraram de outras cidades. O município pesquisado possui 28.774 mil $(13,7 \%)$ pessoas com mais de 60 anos. Emrazão da grande demanda, e considerando os aspectos na transição demográfica que está ocorrendo, percebeu-se a necessidade de maior número de vagas nas instituições de longa permanência do município; atualmente uma das instituições pesquisadas possui 60 idosas na fila de espera feminina.

\begin{tabular}{|c|c|c|c|}
\hline Variável & Categorias & Ocorrência & $\%$ \\
\hline \multirow{7}{*}{ Frequênci } & Semanal & 04 & 13 \\
\hline & Quinzenal & 07 & 24 \\
\hline & & & \\
\hline & Mensal & 04 & 13 \\
\hline & $>1$ mês & 12 & 40 \\
\hline & $>1$ ano & 03 & 10 \\
\hline & TOTAL & 30 & 100 \\
\hline
\end{tabular}

Figura 3 - Distribuição percentual dos entrevistados que recebem visitas. Araraquara, 2011.

Fonte: Dados de pesquisa. 
FARIA et al.



\section{Distribuição dos idosos \\ que recebem visitas (\%)}

Figura 4 - Distribuição percentual da frequência das visitas aos idosos em instituições de longa permanência. Araraquara, 2011.

Fonte: Dados de pesquisa.

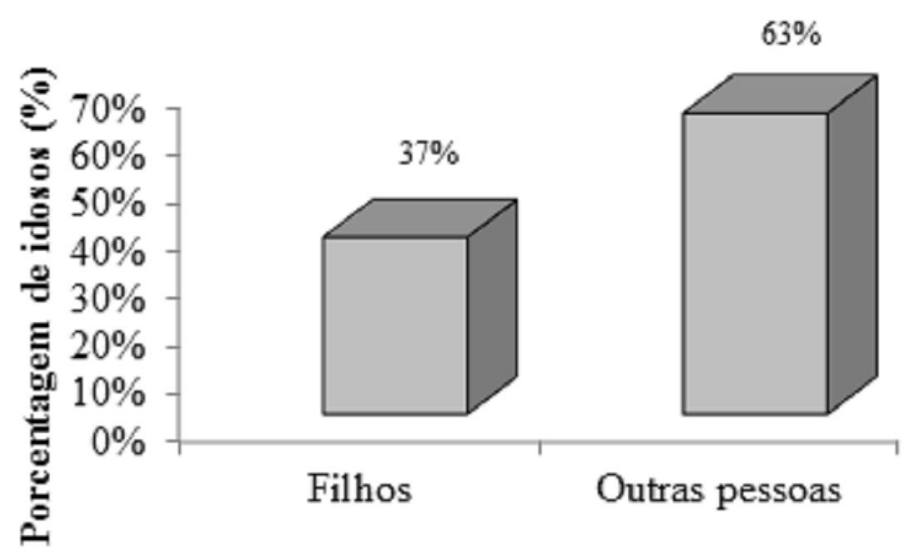

Vinculo dos visitantes

Figura 5 - Distribuição percentual dos idosos entrevistados segundo vínculo dos visitantes. Araraquara, 2011.

Fonte: Dados de pesquisa.

No que se refere ao recebimento de visitas, $64 \%$ dos idosos entrevistados $(\mathrm{n}=30)$ relataram receber visitas; em contraposição, $36 \%(n=17)$ citam ausência de visitas. Com relação ao vínculo dos visitantes, $37 \%$ $(n=11)$ relatam ser os filhos visitantes e $63 \%(n=19)$ outras pessoas, incluindo, principalmente: primos, netos, sobrinhos e amigos. No que se refere à frequência das visitas, $26 \%$ dos idosos entrevistados $(n=12)$ relataram receber visitas no período superior a 1 mês; $15 \%(\mathrm{n}=07)$, quinzenalmente. Houve o mesmo resultado nas variáveis semanal e mensal, com $9 \%$ ( $\mathrm{n}=$ 04). E a variável maior que 1 ano foi escolhida por $5 \%$ dos entrevistados $(n=03)$.

A Figura 1 afirma que $51 \%$ dos idosos têm filhos; no entanto, apenas $37 \%$ visitam seus pais.

As famílias deixam de cuidar dos seus membros 
idosos, o que leva ao aumento dos idosos institucionalizados. Além disso, a convivência cotidiana familiar de origem, constituída por pais e irmãos, tornase, na maioria das vezes, esporádica (BORGHI; MOLITERNO; CARREIRA, 2010).

A falta do apoio familiar no dia-a-dia dos idosos tem maior implicação quando está relacionada à qualidade de vida dos mesmos, pois, após se adaptarem à nova situação, o nível de estresse pode ser reduzido. No entanto, aspectos emocionais e afetivos sofrem grandes consequências negativas; apesar de, na maioria dos casos, os idosos considerarem que constituíram uma nova família, foi evidenciado que sentem falta do apoio dos membros de sua família (AREIAS; LIPP, 2008).

De acordo com a Figura 2, observa-se que $83 \%$ dos idosos residiam no município pesquisado antes da institucionalização, o que sugere a constituição de seu núcleo familiar no local, ou seja, seus familiares residem na mesma cidade. No entanto, $36 \%$ dos entrevistados não recebem nenhum tipo de visita.

Os dados da Figura 5 apontam que $37 \%$ referem receber visitas de seus filhos. No entanto, $63 \%$ dos visitantes são outras pessoas. Ao comparar os resultados, na Figura 1, 51\% dos idosos entrevistados possuem filhos. Por que esses filhos não visitam seus pais? Essa questão oferece margens para profunda reflexão e a elaboração de novas pesquisas que contribuam para a melhoria da qualidade de vida dos núcleos familiares de idosos institucionalizados e o reforço do relacionamento interpessoal entre os pais e filhos.

\section{Conclusão}

Para a pessoa idosa institucionalizada, o abandono e a solidão podem ser refletidos pela falta de visitas de seus familiares. Certamente o achado mais importante neste estudo, além da corroboração da multiplicidade de perdas presentes na história de vida dos idosos institucionalizados, foi o sentimento de abandono inerente a todos esses idosos, tanto familiar quanto da sociedade em geral.

Pode-se considerar que os idosos são seres humanos vivenciando a última fase de seu desenvolvimento, marcada inegavelmente mais por perdas que por ganhos. Durante o processo de institucionalização, sua individualidade pode ser comprometida, visto que o idoso perde o poder de gerir sua própria vida, sua autonomia ao não poder sair da instituição quando deseja, não administra seu dinheiro e quase sempre não possui roupas e pertences pessoais, já que estes são compartilhados.

Viver dignamente é a forma mais sublime que podemos proporcionar à pessoa idosa, enquanto profissionais da área da saúde onde impere o respeito, a gratidão, a valorização ao assumirmos a responsabilidade pelo seu cuidado e proporcionar momentos felizes, singulares e inéditos.

Maior atenção pelos profissionais da saúde que atendem a pessoa idosa em implantar ações, a fim de resgatar o vínculo familiar nesta fase de finitude e que deveria ser brindada com grande júbilo, principalmente com aqueles a que eles dedicaram toda sua vida: seus filhos.

\section{REFERÊNCIAS}

AREIAS, G. L.; LIPP, M. E. N. Comparação do nível de stress entre idosos que moram com a família e aqueles que vivem em casa de repouso. In: ENCONTRO DE INICIAÇÃO CIENTÍFICA DA PUC, 13 -Campinas, 2008.

BOFF, L. Saber cuidar: ética do humanocompaixão pela Terra. Petrópolis: Vozes, 1999.

BORGHI, A. C.; MOLITERNO, A. C. M; CARREIRA, L. Caracterização dos idosos institucionalizados no asilo São Vicente de Paulo de Maringá. In: SIMPÓSIO MARINGAENSE DE GERONTOLOGIA, 2. 2010. Disponível em: <http:// www.uem.br/unati/images/stories/unati/anais2010/ anais_unati2010_11_01_2010_versao_final.pdf>. Acesso em: 07 agosto 2011.

BORN, T.; BOECHAT, N. S. Aqualidade dos 
cuidados ao idoso institucionalizado. In: FREITAS, E. V.; PY, L.; CANÇADO, F. A. X.; GORZONI, M. L. Tratado de Geriatria e Gerontologia. 2 ed. Rio de Janeiro: Guanabara Koogan, 2006. Cap. 119, p. 1131-1141.

\section{BRASIL. MINISTÉRIO DA SAÚDE. AGENCIA} NACIONAL DE VIGILÂNCIA SANITÁRIAANVISA. Resolução da Diretoria Colegiada n 283. Regulamento Técnico que define normas de funcionamento para as Instituições de Longa Permanência para Idosos. 2005. Disponível em: <http://www.saude.sp.gov.br/atos_normativos/ legislacao-sanitaria/estabelecimentos-de-saude/ atencao-ao-idoso/RES_283.pdf>. Acesso em: 20 abril 2011.

CHAIMOWICZ, F. Dinâmica da institucionalização de idosos em Belo Horizonte, Brasil. Revista Saúde Pública, v. 33, p. 454-460, 1998.

\section{INSTITUTO BRASILEIRO DE GEOGRAFIA E} ESTATÍSTICA - IBGE. Coordenação de população e indicadores sociais. Síntese de indicadores sociais: um a análise das condições de vida da população brasileira: 2011. Rio de Janeiro.

Disponível em: http://www.ibge.gov.br/home/ estatistica/populacao/censo2010/default.shtm. Acesso em: 10 agosto 2011.
PAPALEO, M. Gerontologia: a velhice e o envelhecimento em visão globalizada. São Paulo: Editora Atheneu, 2005.

PAVAN, F. J.; MENEGHEL, S. N.; JUNGES, J. R. Mulheres idosas enfrentando a institucionalização.

Cad. Saúde Pública, Rio de Janeiro, set. 2008.

Disponível em: http://www.scielosp.org/ scielo.php?script=sci_arttext\&pid=S0102311X2008. Acesso em: 17 fevereiro 2011.

PERLINI, N. M. O. G.; LEITE, M. T.; FURINI, A. C. Em busca de uma instituição para a pessoa idosa morar: motivos apontados por familiares. Revista da Escola Enfermagem da USP, São Paulo, v. 41, n. 2, p. 229-236, 2007.

PINHEIRO, L.; GALIZA, M.; FONTOURA, N. Novos arranjos familiares, velhas convenções sociais de gênero: a licença parental como política pública para lidar com essas tensões. Revista Estudos Feministas, Florianópolis, v. 17, n. 3, p. 851-859, set./dez. 2009.

REIS, L. A.; TORRES, G. V.; REIS, L. A.; FERNANDES, M. H. Características sóciodemográficas e de saúde de idosos dependentes residentes em domicílio. Revista Espaço para a Saúde, v. 10, n. 2, p. 12-17, 2008. 